\title{
Cambial Variants Combine Successive Cambia and Intraxylary Phloem in Convolvulaceae in Taiwan
}

\author{
Sheng-Zehn Yang1* ${ }^{*}$ Po-Hao Chen ${ }^{2}$, Chien-Fan Chen ${ }^{3}$ \\ ${ }^{1}$ Department of Forestry, National Pingtung University of Science and Technology, Taiwan \\ ${ }^{2}$ Graduate Institute of Bioresources, National Pingtung University of Science and Technology, Taiwan \\ ${ }^{3}$ Taiwan Forestry Research Institute, Taiwan \\ Email: ^yangsz@mail.npust.edu.tw, 126571583@yahoo.com.tw, chenc@tfri.gov.tw
}

How to cite this paper: Yang, S.-Z., Chen, P.-H. and Chen, C.-F. (2020) Cambial Variants Combine Successive Cambia and Intraxylary Phloem in Convolvulaceae in Taiwan. American Journal of Plant Sciences, 11, 437-453.

https://doi.org/10.4236/ajps.2020.113032

Received: February 4, 2020

Accepted: March 23, 2020

Published: March 26, 2020

Copyright $\odot 2020$ by author(s) and Scientific Research Publishing Inc. This work is licensed under the Creative Commons Attribution International License (CC BY 4.0).

http://creativecommons.org/licenses/by/4.0/

(c) (i) Open Access

\begin{abstract}
Secondary stem growth develops different cambial variants in Convolvulaceae. Here, the cambial variant features of 17 species in seven genera (Convolvulaceae) in Taiwan are presented. The xylem rings produce various successive cambial types and the primary xylem and intraxylary phloem are produced in the outer and inner pith, respectively. The two Argyreia species have round or elliptical stems with continuous secondary xylem and phloem rings. In the eight Ipomoea species, secondary growth has two to four layers of successive cambia and xylem, dispersed with parenchyma tissue, except for I. nil and $I$. violacea with one layer of successive cambia. The first secondary xylem segments are separated by few or many xylem rays: $I$. cairica and $I$. $o b$ scura have three to five xylem rays and $I$. triloba has numerous xylem rays. The first secondary xylem of Erycibe henryi is divided by numerous rays in small stems, but develops more layers, as adjacent segments separated by xylem rays, in larger stems. Owing to continuous vascular bundle division, it has a dissected xylem. The secondary growth of Distimate tuberosa and Operculina turpethum produces two layers of successive cambia; the secondary successive cambium is divided evenly by rays into many arcs/segments. The stem thickness of $I$. pes-caprae subsp. brasiliensis increases via concentric successive cambia. Owing to shallowly lobed stems with directional successive cambia, I. obscura and I. triloba stems are irregularly shaped. I. littoralis and Stictocardia tilifolia stems are triangular without directional successive cambia. I. hederifolia and Merremia gemella stems have two to three deep lobes. Parenchyma proliferation results in thicker cortex with mucilaginous canals. The xylem vessels are dispersed and diffuse-porous; the vasicentric paratracheal parenchyma around them is common to all species. A bracketed key was developed comparing the secondary xylem structures of the 17 species, providing a basis for further morphological studies.
\end{abstract}




\section{Keywords}

Arcs/Segments, Convolvulaceae, Dissected Xylem, Lobe Stem

\section{Introduction}

The vascular cambium of climbing plants is usually located around the circumference of the stem, and if the activity of the cambium is not continuous, some of the circumferential regions produce tissue that is very different from the adjacent areas. Such irregular growth may be associated with the cambium or with morphogenetic factors derived from the cambium that affect cell activity [1]. Under normal conditions, each cambium produces xylem centripetally and phloem centrifugally, but both types of tissue have large amounts of parenchyma cells. All parenchyma cells in climbing plants can be redifferentiated into meristematic cells, which may give rise to vascular bundles, cork cambia, dilatation tissue, or adventitious buds [1]. The stem cross-section generally changes from a regular circular to an irregular shape after secondary growth, owing to the uneven deposition of secondary xylem and the eccentricity of successive cambia [2]. As the stem twines around a support, cambial activity increases the amount of xylem and phloem on the opposite side of the stem [3].

Cambial variants are divided into two types: those that originate from a single cambium and those that originate from multiple cambia [4]. Within Convolvulaceae, several cambial variants have been reported, such as successive cambia, interxylary phloem, xylem parts dispersed by parenchyma, and intraxylary phloem [4] [5] [6] [7] [8]. The successive cambia are divided into concentric bands and non-concentric bands [9]. The activity of concentric bands produces vascular bundles of the same width, and parenchyma cells with alternating, well-defined xylem and phloem rings; the pith is usually located in the center. The activity of non-concentric bands produces an asymmetric band in different directional successive cambia, leading to intensified development of one to three sides of the stem and an off-center pith [10].

The genus Argyreia develops successive cambia [11], medullary bundles, interxylary phloem, and intraxylary phloem [12] [13]. The anatomy of several species of Argyreia has been studied, including the structure of the medullary bundles in $A$. roxburghii (Wall.) Arn. ex Choisy [12] and the tri-lobed stem and successive cambia in $A$. hookeri C. B. Clarke [8].

The genus Ipomoea has successive cambia, interxylary phloem, and fibrous woody parts dispersed by parenchyma proliferation [11]. In an early stage of primary growth, some Ipomoea species stems form primary xylem [14]. Under normal developmental conditions, the vascular bundles in the genus Ipomoea usually develop precisely in their positions. Initially, the secondary growth of Ipomoea is normal, producing both xylem and phloem. This is followed by the activity of additional cambia, each forming around a single vascular bundle or 
group of vascular bundles, producing both xylem and phloem.

Lowell and Lucansky [15] reported that the primary growth of Ipomoea hederifolia L. comprises a bicollateral bundle stage and a cambium-like meristem stage, whereas the secondary growth consists of a normal cambial stage, an anomalous stage, and a supernumerary cambial stage. Rajput et al. [16] indicated that the discontinuous segments of the secondary xylem in the second ring in $I$. hederifolia showed xylem with reverse orientation, producing secondary xylem centrifugally and secondary phloem centripetally. Patil et al. [17] also showed that one of the successive cambial layers develops anomalous cambial arcs/segments with reverse orientation in $I$. hederifolia. The stem thickness of Ipomoea obscura (L.) Ker Gawl. and Ipomoea pes-caprae (L.) R. Br. in cross-section consist of three to four layers of secondary xylem and alternating phloem rings, giving rise to multiple/successive cambia [2] [18]. The stem of $I$. quamoclit L. subsequently develops lobed, concentric, and diffuse (foraminate) anomalous secondary growth [19]; and I. turbianata Lag. forms internal cambium and intraxylary phloem [7].

Convolvulaceae comprises approximately 53 genera and 1660 species distributed worldwide [20]. Of these, 14 genera and 44 species of Convolvulaceae, mostly lianas, have been recorded in Taiwan [21]. From 2000 to 2017, 14 species were published in Taiwan, including one new species of the genus Argyreia [22] and 13 naturalized species, including two species of the genus Cuscuta [23], five species of the genus Ipomoea [24] [25] [26] [27] [28], one species of the genus Evolvulus [29], four species of the genus Merremia [30] [31] [32] [33], and one species of the genus Jacquemontia [34]. Thus, there are approximately 14 genera and 58 species of Convolvulaceae in Taiwan.

Multiple types of cambial variants are found within Convolvulaceae, but the growth patterns of the stem cross-sections of many members of Convolvulaceae in Taiwan have not been described. Various stem structures and identifications have been reported in the Menispermaceae and Fabaceae families in Taiwan [35] [36]. In the present study, we examined the stem cross-sections of Convolvulaceae vines in Taiwan, and how the cambial variants combined with successive cambia and intraxylary phloem. As cambial variations constitute an extremely diverse morphology, the present study attempts to 1) provide detailed photographs of the features discussed and 2) provide a bracketed key based on the anatomical characteristics of the stems to facilitate the identification of irregular cambial activity in the Convolvulaceae family in Taiwan.

\section{Materials and Methods}

\subsection{Research Materials}

The species datasets included species scientific name, collector, herbarium and voucher number, and collection locality. Cambial variations in the investigated species were used to construct a comparison table. Multiple samples of each species were collected from 2017 to 2019 in Taiwan and stems with thick bark and 
visible secondary growth characteristics in their cross-sections were selected in the field. Two stem sizes of five species were collected to examine cambial variations related to different stem sizes.

To keep the material fresh and retain humidity, the stems were stored in the collecting bag. One or two samples with obvious and easy-to-observe cambial variations were selected per species for photographs and the scoring of morphological characters.

\subsection{Research Methods}

In the laboratory, the fresh stems were cut into pieces approximately $5 \mathrm{~cm}$ long, and a freehand cross-section of each stem was made with a razor blade. The stem cross-section was immediately photographed using a Nikon D7100 SLR digital camera with 1:1 lens (Lens AF Micro Nikon 60 mm 1:2.8D; Nikon Corporation, Tokyo, Japan). All specimens were oven-dried at $60^{\circ} \mathrm{C}$ for $4-5$ days and then stored at $-20^{\circ} \mathrm{C}$ for $3-4$ days, and deposited in the Provincial Pingtung Institute (PPI) herbarium at the National Pingtung University of Science and Technology, Pingtung, Taiwan, for subsequent identification. The nomenclature follows Flora of Taiwan Volume 4 [21] with the dissolution of the Convolvulaceae tribe Merremieae, and the new classification of the constituent genera [37].

The morphological characteristics of the irregular stem structures of each species were investigated as followed: stem diameter $(\mathrm{mm})$, shape of the fresh stem cross-section, number of successive cambium layers, segment number of the first secondary xylem, minimum and maximum diameter of vessels $(\mu \mathrm{m})$, mean diameter of vessels $(\mu \mathrm{m})$ (mean \pm standard deviation, $\mathrm{SD}, \mathrm{n}=25$ ), directional successive cambia (+/-), cambial arcs/segment (+/-), and cork (+/-). Stem diameter was assessed using the Radford [38] definition, according to the plan length/width proportion. Vessels with two distinct diameter classes, and wood that was not ring-porous, were measured using the larger size class, and 25 measured vessels (mean $\pm \mathrm{SD}$ ) are required by the definition of Wheeler et al. [39]. Quantitative anatomical traits such as the diameter of the stems (length and width, $\mathrm{mm} \times \mathrm{mm}$ ) or minimum and maximum sizes of the xylem vessels were determined using Image-J software [40]. The diameter of nearly circular stems was measured from the average of four diagonal lines; whereas non-circular stems were measured using the minimum and maximum diameter as length and width.

\section{Results}

\subsection{Cambial Variants Photographs and Explanation}

Cambium variations in stems, combined with successive cambia and intraxylary phloem, were assessed in approximately 17 species in seven genera in the Convolvulaceae family in Taiwan. The dataset of these species is provided in Table 1. Two species, A. akoensis S. Z. Yang, P. H. Chen \& Staples and A. formosana Ishigami ex T. Yamaz., are endemic and distributed at low elevations in southern 
Table 1. The seven genera and 17 species of Convolvulaceae in Taiwan referred to in this study.

\begin{tabular}{|c|c|c|c|}
\hline Species & Collector & $\begin{array}{l}\text { Herbarium and } \\
\text { voucher number }\end{array}$ & Collection localities \\
\hline $\begin{array}{l}\text { Argyreia akoensis S. Z. } \\
\text { Yang, P.H. Chen \& Staples }\end{array}$ & Chen, Po-Hao & PPI 77258 & $\begin{array}{l}\text { Liangshan, Machia, } \\
\text { Pingtung County }\end{array}$ \\
\hline $\begin{array}{l}\text { Argyreia formsana } \\
\text { Ishigami ex T. Yamaz. }\end{array}$ & Yang, Sheng-Zehn & PPI 57191 & $\begin{array}{l}\text { Lilongshan, Shlzi, } \\
\text { Pingtung County }\end{array}$ \\
\hline $\begin{array}{l}\text { Distimate tuberosa (L.) } \\
\text { Simoes \& Staples }\end{array}$ & Chen, Chien-Fan & PPI 63209 & Liu-kuei, Kaohsiung City \\
\hline Erycibe henryi Prain & Yang, Sheng-Zehn & PPI 68829 & $\begin{array}{l}\text { Tajen Hsiang, } \\
\text { Taitung County }\end{array}$ \\
\hline Ipomoea alba $\mathrm{L}$. & Chen, Po-Hao & PPI 77479 & Gaoshu, Pingtung County \\
\hline Ipomoea cairica (L.) Sweet & Lo, Tin-Yan & PPI 69043 & $\begin{array}{l}\text { Liugiuyu, Liugiu, } \\
\text { Pingtung County }\end{array}$ \\
\hline Ipomoea hederifolia $\mathrm{L}$. & Chen, Po-Hao & PPI 76939 & $\begin{array}{c}\text { Chiahsien, Paiyunshan, } \\
\text { Kaohsiung City }\end{array}$ \\
\hline $\begin{array}{l}\text { Ipomoea indica } \\
\text { (Burm.) Merr. }\end{array}$ & $\begin{array}{l}\text { Shiu, Sneng-Yuan } \\
\text { Wu, Jia-Jin }\end{array}$ & PPI 72827 & Ranai, Nantou County \\
\hline Ipomoea littoralis Blume & Hsiuh, Guang-Pu & PPI 63597 & $\begin{array}{l}\text { Gongguan, Ludao, } \\
\text { Taitung County }\end{array}$ \\
\hline $\begin{array}{l}\text { Ipomoea obscura } \\
\text { (L.) Ker Gawl. }\end{array}$ & Chen, Po-Hao & PPI 75775 & Paili, Pingtung County \\
\hline Ipomoea nil (L.) Roth & Kuo, Shium-Ming & PPI 55342 & Neipu, Pingtung County \\
\hline $\begin{array}{l}\text { Ipomoea pescaprae (L.) } \\
\text { R. Br. subsp. brasiliensis } \\
\text { (L.) Ooststr. }\end{array}$ & Liang, Ju-Snuo & PPI 73457 & $\begin{array}{l}\text { Mianhua Island, } \\
\text { Keelung, Taipei }\end{array}$ \\
\hline Ipomoea triloba $\mathrm{L}$. & Chen, Po-Hao & PPI 75751 & $\begin{array}{l}\text { Lilongshan, Shlzi, } \\
\text { Pingtung County }\end{array}$ \\
\hline Ipomoea violacea $\mathrm{L}$. & Chen, Po-Hao & PPI 77453 & $\begin{array}{l}\text { Liugiuyu, Liugiu, } \\
\text { Pingtung County }\end{array}$ \\
\hline $\begin{array}{l}\text { Merremia gemella } \\
\text { (Burm. f.) Hallier f. }\end{array}$ & Chen, Po-Hao & PPI 75756 & $\begin{array}{c}\text { Lilungshan, } \\
\text { Pingtung County }\end{array}$ \\
\hline $\begin{array}{l}\text { Operculina turpethum } \\
\text { (L.) S. Manso }\end{array}$ & Yang, Sheng-Zehn & PPI 66248 & $\begin{array}{l}\text { Hsiaoliuchiu, } \\
\text { Pingtung County }\end{array}$ \\
\hline $\begin{array}{l}\text { Stictocardia tiliifolia } \\
\text { (Desr.) Hallier } \mathrm{f} .\end{array}$ & Chen, Po-Hao & PPI 77154 & $\begin{array}{l}\text { Shipa Roheinshan, } \\
\text { Kaohsiung City }\end{array}$ \\
\hline
\end{tabular}

Taiwan. The anatomical and morphological details of the stems assessed in this study are listed (Table 2) and presented in Figures 1-5. In all 17 species, the stem cross-section was formed from mucilaginous canals, vasicentric parenchyma, and strands of intraxylary phloem. The intraxylary phloem strands were located on the inner margin of the pith (Figure 1(b), Figure 1(d), Figure 1(f), Figure 2(d), Figure 3(b), Figure 3(e), Figures 4(a)-(d)).

The epidermis is one of the diagnostic features of these species. Thus, a noticeable cork (phellem) was observed on the larger stems of $A$. formosana (Figure 1(c)), but absent in $A$. akoensis. Among the 10 Ipomoea species, five species also 
Table 2. Morphological characteristics of stem cross-sections showing the successive cambia and intraxylary phloem of 17 species (Convolvulaceae) in Taiwan.

\begin{tabular}{|c|c|c|c|c|c|c|c|c|c|}
\hline $\begin{array}{c}\text { Characters } \\
\text { Scientific name }\end{array}$ & $\begin{array}{c}\text { Stem diameter } \\
(\text { length } \times \text { width }) \\
(\mathrm{mm})\end{array}$ & $\begin{array}{l}\text { Fresh stem } \\
\text { shape }\end{array}$ & $\begin{array}{c}\text { Number of } \\
\text { successive } \\
\text { cambia layers }\end{array}$ & $\begin{array}{l}\text { Segment number } \\
\text { of the first } \\
\text { secondary xylem }\end{array}$ & $\begin{array}{c}\text { Min-max vessels } \\
\text { diameter } \\
(\mu \mathrm{m})\end{array}$ & $\begin{array}{c}\text { Mean diameter of } \\
\text { vessels }(\text { mean } \pm \\
\mathrm{SD}, \mathrm{n}=25)(\mu \mathrm{m})\end{array}$ & $\begin{array}{c}\text { Directional } \\
\text { successive } \\
\text { cambia } \\
(+/-)\end{array}$ & $\begin{array}{c}\text { Cambial } \\
\text { arc/ } \\
\text { segment } \\
(+/-)\end{array}$ & $\begin{array}{l}\text { Cork } \\
(+/-)\end{array}$ \\
\hline Argyreia akoensis & $4.2 \times 4.0,9.5 \times 9.4$ & round & $2-3$ & 3 & $47-139 ; 134-299$ & $79 \pm 21 ; 198 \pm 47$ & - & - & - \\
\hline Argyreia formsana & $4.2 \times 4.2,5.0 \times 4.8$ & elliptic & 2 & 3 & $65-149 ; 61-199$ & $100 \pm 23 ; 124 \pm 35$ & - & - & + \\
\hline Distimate tuberosa & $39.4 \times 32.4$ & oblong & 2 & 3 & $238-459$ & $336 \pm 55$ & - & + & - \\
\hline Erycibe henryi & $7.2 \times 7.0,18.6 \times 17.1$ & round & $3-4$ & numerous & $63-123 ; 101-228$ & $142 \pm 31 ; 91 \pm 17$ & - & + & - \\
\hline Ipomoea alba & $18.5 \times 12.6$ & irregular & 2 & 3 & $109-302$ & $206 \pm 50$ & - & - & - \\
\hline Ipomoea cairica & $17.9 \times 15.9$ & irregular & 3 & 5 & $128-302$ & $178 \pm 39$ & - & + & + \\
\hline Ipomoea hederifolia & $5.5 \times 2.4,6.4 \times 5.7$ & 2-3 lobed & $2-3$ & $2-3$ & $75-177 ; 61-158$ & $123 \pm 27 ; 117 \pm 27$ & + & + & - \\
\hline Ipomoea indica & $11.0 \times 7.3$ & irregular & 2 & 5 & $72-228$ & $137 \pm 34$ & - & - & - \\
\hline Ipomoea littoralis & $5.3 \times 5.2$ & triangular & 2 & 3 & $83-231$ & $149 \pm 35$ & - & - & + \\
\hline Ipomoea nil & $7.4 \times 7.3$ & round & 1 & numerous & $104-226$ & $157 \pm 35$ & - & - & - \\
\hline Ipomoea obscura & $12.3 \times 10.4$ & irregular & 4 & 4 & $40-105$ & $70 \pm 16$ & + & + & + \\
\hline $\begin{array}{l}\text { Ipomoea pescaprae } \\
\text { subsp. brasiliensis }\end{array}$ & $17.5 \times 17.2$ & oblong & 3 & numerous & $82-245$ & $151 \pm 36$ & - & + & + \\
\hline Ipomoea triloba & $6.8 \times 4.3,10.3 \times 9.4$ & irregular & $2-4$ & numerous & $94-175 ; 39-157$ & $143 \pm 20 ; 83 \pm 37$ & + & + & + \\
\hline Ipomoea violacea & $7.5 \times 7.4$ & oblong & 1 & 2 & $64-272$ & $170 \pm 51$ & - & - & - \\
\hline Merremia gemella & $4.4 \times 2.0$ & tri-lobed & 2 & 3 & $46-215$ & $127 \pm 37$ & + & - & - \\
\hline $\begin{array}{l}\text { Operculina } \\
\text { turpethum }\end{array}$ & $29.9 \times 26.7$ & round & 3 & 3 & $104-295$ & $198 \pm 46$ & - & + & - \\
\hline Stictocardia tiliifolia & $7.9 \times 6.6$ & triangular & 2 & $4-5$ & $125-247$ & $182 \pm 33$ & - & - & - \\
\hline
\end{tabular}

Note: +/-: present/absent.

developed cork (Table 2). The primary growth of the stem was similar among all the species-a cambium-like meristem was formed, and intraxylary phloem and primary xylem were produced in the inner and outer pith respectively. The secondary growth developed one to four successive cambial rings among the studied species. The successive rings of the secondary xylem alternate with phloem rings (Figure 1(b), Figure 2(d), Figure 2(e), Figures 3(c)-(f)). The first layer of the successive cambium developed normal secondary tissue, which produced secondary xylem centripetally and secondary phloem centrifugally in all species (Figures 1-4). The second or third layers of secondary tissue developed from the anomalous cambial stage to the supernumerary cambial stage, forming a ring of small cambial arcs/segments (Figure 1(e), Figure 2(a), Figures 2(c)-(e), Figure 3(c), Figure 3(d), Figure 3(f), Figure 4(c), Figure 4(d)). These small cambial arcs/segments were fused to a continuous cambial ring and the adjacent segments separated by rays. The supernumerary cambial stage in the first secondary growth of the stem formed a discontinuous xylem ring generally separated by a few wide rays (Figure 1(a), Figure 1(c), Figure 1(e), Figure 2(a), Figure 2(b), Figure 2(d), Figure 2(f), Figures 3(a)-(c)). However, the xylem rings of Erycibe 


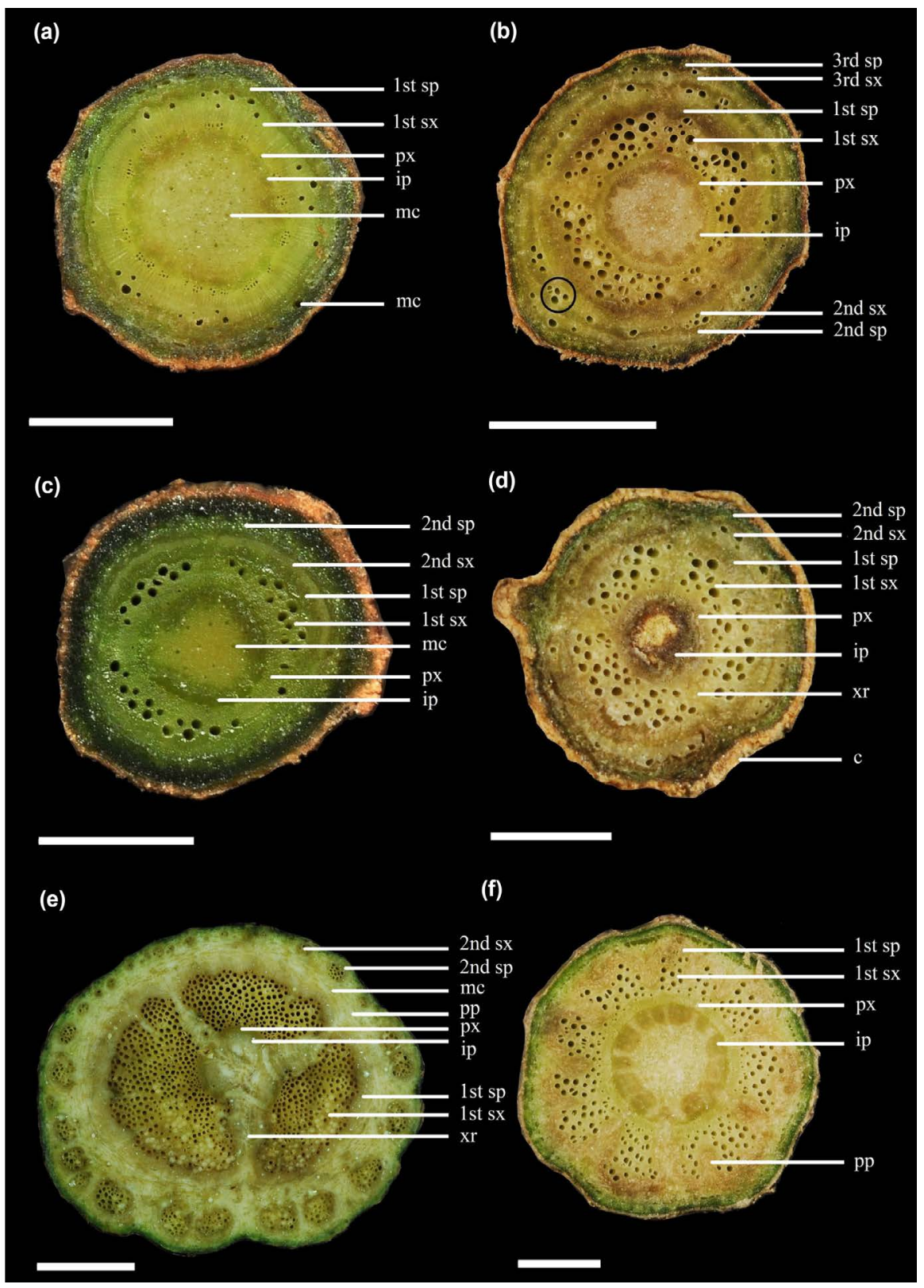

Figure 1. Stem cross-sections of Argyreia, Distimate, and Erycibe (Convolvulaceae). (a)-(b) Argyreia akoensis. (a) Small stem round in shape. (b) Large stem showing vasicentric paratracheal parenchyma (circle). (c)-(d) Argyreia formsana. (d) Thick stem showing obvious cork. (e) Distimate tuberosa. (f) Erycibe henryi. Abbreviations: 1st sx (first secondary xylem), 1st sp (first secondary phloem), 2nd sx (second secondary xylem), 2nd sp (second secondary phloem), 3rd sx (third secondary xylem), 3rd sp (third secondary phloem), c (cork), ip (intraxylary phloem), mc (mucilaginous canal), px (primary xylem), $x r$ (xylem ray). Scale bars: (a), (c), (d), (f) $=2 \mathrm{~mm}$; (b) $=5 \mathrm{~mm}$, (e) $=10 \mathrm{~mm}$.

henryi (Figure 3(a)), Ipomoea nil (Figure 3(b)), I. pes-caprae subsp. brasiliensis (Figure 3(d)), and I. triloba (Figure 3(e), Figure 3(f)) were separated by several thin rays. Parenchyma proliferation is often present in some species, which results in thick stems with mucilaginous canals (Figure 2(b), Figure 2(c), Figure 2(f), Figure 3(a), Figure 3(c), Figure 4(a)). 


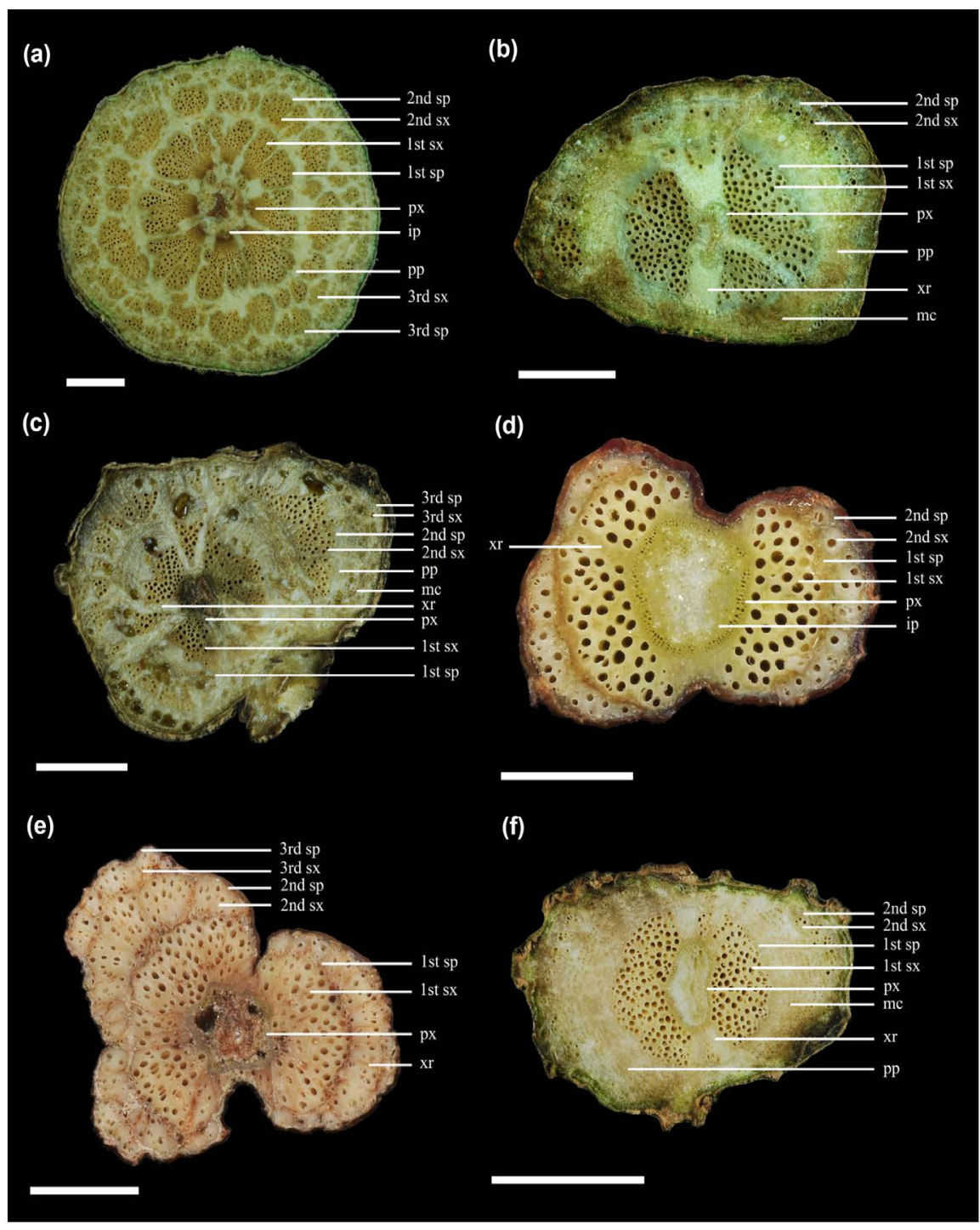

Figure 2. Stem cross-sections of Erycibe and Ipomoea (Convolvulaceae). (a) Erycibe henryi. The secondary xylem rings are divided by xylem rays and form an obviously dissected type of xylem. (b) Ipomoea alba. (c) Ipomoea cairica. (d)-(e) Ipomoea hederifolia. d. Young stem showing a bi-lobed shape owing to two segments of functionally slow cambium. (f) Ipomoea indica. Abbreviations: 1st sx (first secondary xylem), 1st sp (first secondary phloem), 2nd sx (second secondary xylem), 2nd sp (second secondary phloem), 3rd sx (third secondary xylem), 3rd sp (third secondary phloem), ip (intraxylary phloem), mc (mucilaginous canal), pp (parenchyma proliferation), px (primary xylem), xr (xylem ray). Scale bars: (a), (b), (f) $=5 \mathrm{~mm}$; (c)-(e) $=2 \mathrm{~mm}$.

Five species, A. akoensis, A. formosana, E. henryi, I. hederifolia, and I. triloba L. were collected to investigate the differences in cambial variations related to different stem sizes. A. akoensis formed its first layer of secondary tissue when the diameter of the stem was $4.2 \times 4.0 \mathrm{~mm}$ (Figure 1(a)), and developed its third layer of secondary tissue (Figure 1(b)) when the diameter of the stem was ca. 9.5 $\times 9.4 \mathrm{~mm}$. In contrast, $A$. formosana showed little difference in stem diameter, although it developed second secondary tissue (Figure 1(c), Figure 1(d); Table 2). 


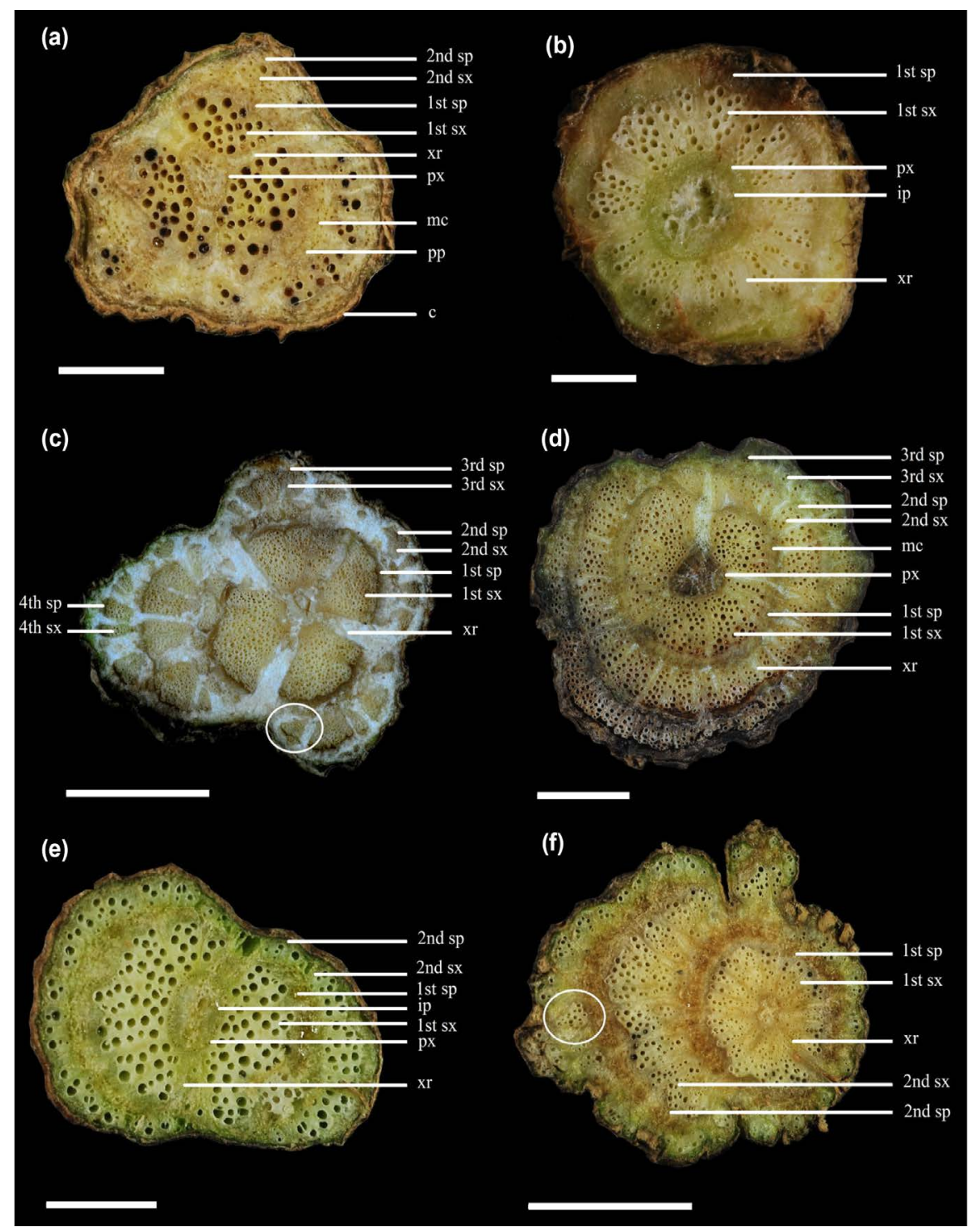

Figure 3. Stem cross-sections of Ipomoea (Convolvulaceae). (a) Ipomoea littoralis, stem triangular in shape. (b) Ipomoea nil, forms only one layer of secondary xylem. (c) Ipomoea obscura, secondary xylem divided by xylem rays to form a ring of small cambial arcs/segments (circle). (d) Ipomoea pes-caprae subsp. brasiliensis, one to three secondary xylems evenly divided by numerous rays. (e)-(f) Ipomoea triloba. f. Directional successive cambium and a ring of small cambial arcs/segments (circle). Abbreviations: 1st sx (first secondary xylem), 1st sp (first secondary phloem), 2nd sx (second secondary xylem), 2nd sp (second secondary phloem), 3rd sx (third secondary xylem), 3rd sp (third secondary phloem), 4th sx (forth secondary xylem), 4th sp (forth secondary phloem), c (cork), ip (intraxylary phloem), mc (mucilaginous canal), pp (parenchyma proliferation), px (primary xylem), xr (xylem ray). Scale bars: (a), (d), (f) $=2 \mathrm{~mm}$; (b), (c), (e) $=5 \mathrm{~mm}$.

The first secondary xylem of $E$. henryi was divided by numerous rays when the diameter of the stem was smaller $(7.2 \times 7.0 \mathrm{~mm})$, and developed more secondary xylem rings, as adjacent arcs/segments separated by xylem rays, when the diameter of the stem was $18.6 \times 17.1 \mathrm{~mm}$. Both I. hederifolia and I. triloba also showed different stem types, with the larger stems developing two to three deep 


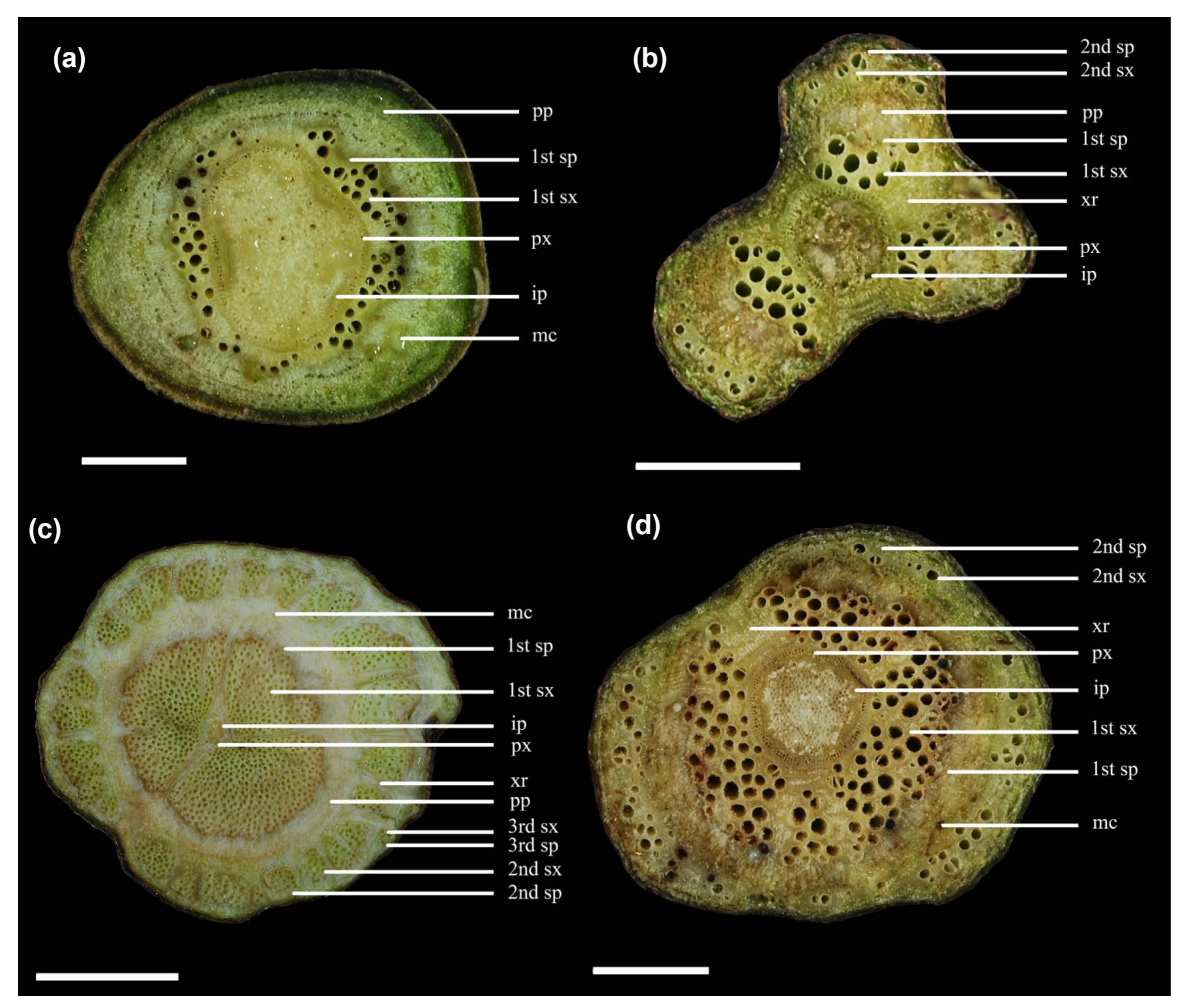

Figure 4. Stem cross-sections of Ipomoea, Merremia, Operculina, and Stictocardia (Convolvulaceae). (a) Ipomoea violacea, formed only one layer of secondary xylem. (b) Merremia gemella, stem tri-lobed in shape. (c) Operculina turpethum, second secondary xylem divided by xylem rays and form a ring of small cambial arcs/segments. (d) Stictocardia tiliifolia, first secondary xylem divided by five xylem rays. Abbreviations: 1st sx (first secondary xylem), 1st sp (first secondary phloem), 2nd sx (second secondary xylem), 2nd sp (second secondary phloem), 3rd sx (second secondary xylem), 3rd sp (second secondary phloem), 4th sx (forth secondary xylem), 4th sp (forth secondary phloem), ip (intraxylary phloem), mc (mucilaginous canal), pp (parenchyma proliferation), px (primary xylem), $x r$ (xylem ray). Scale bars: (a), (d), (f) $=2 \mathrm{~mm}$; (b), (c), (e) $=5$ $\mathrm{mm}$.

lobes (Figure 2(d), Figure 2(e)) and a directional succession cambium (Figure $3(\mathrm{e})$, Figure $3(\mathrm{f})$ ), respectively.

The vessel distribution of the secondary xylem was often diffuse-porous. The species I. obscura had the lowest vessel diameter $(70 \pm 16 \mu \mathrm{m})$ and Distimate tuberosa had the highest vessel diameter $(336 \mu \mathrm{m})$. The vessel diameter of I. triloba differed depending on stem diameter, with larger vessels $(143 \pm 20 \mu \mathrm{m})$ in smaller stems $(6.8 \times 4.3 \mathrm{~mm})$ and smaller vessels $(83 \pm 37 \mu \mathrm{m})$ in larger stems $(10.3 \times 9.4 \mathrm{~mm})$ (Figure 5). The vessel diameters of all species are recorded in Figure 5 and Table 2.

\subsection{Species Characterization}

Based on the characteristics of the stem cross-sections, we created a bracketed key for identification of the 17 climbing species. This key allows species identification when leaves are not available. 

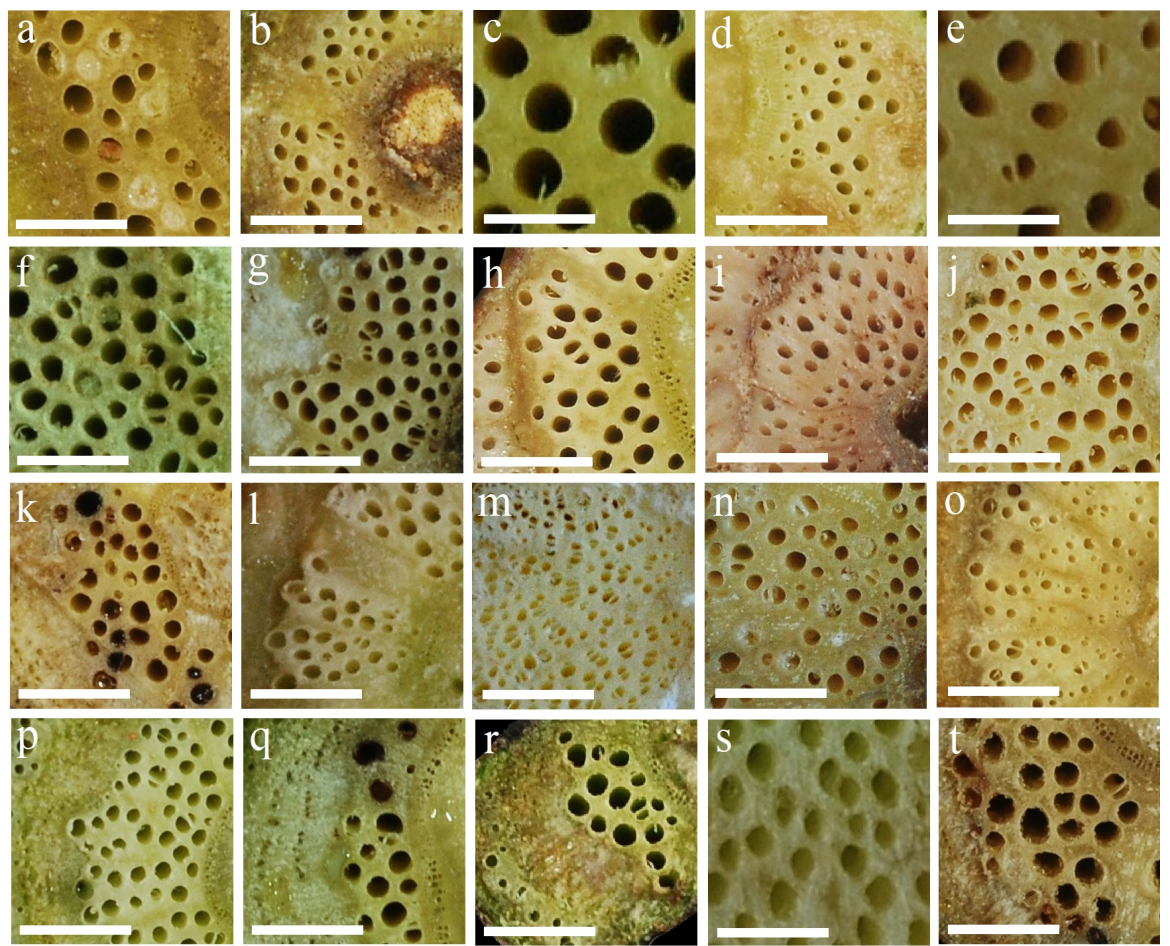

Figure 5. The magnification of stem cross-section of Argyreia, Distimate, Erycibe, Ipomoea, Merremia, Operculina, and Stictocardia (Convolvulaceae) showing the diameter of the vessels (mean \pm SD). (a) Argyreia akoensis (198 $\pm 47 \mu \mathrm{m}$ ). (b) Argyreia formsana (124 $\pm 35 \mu \mathrm{m})$. (c) Distimate tuberosa $(336 \pm 55 \mu \mathrm{m})$. (d), (e) Erycibe henryi $(142 \pm 31 \mu \mathrm{m}$; 91 $\pm 17 \mu \mathrm{m})$. (f) Ipomoea alba $(206 \pm 50 \mu \mathrm{m})$. (g) Ipomoea cairica $(178 \pm 39 \mu \mathrm{m})$. (h)-(i) Ipomoea hederifolia $(123 \pm 27 \mu \mathrm{m} ; 117 \pm 27 \mu \mathrm{m})$. (j) Ipomoea indica $(137 \pm 34 \mu \mathrm{m})$. (k) Ipomoea littoralis $(149 \pm 35 \mu \mathrm{m})$. (l) Ipomoea nil $(157 \pm 35 \mu \mathrm{m})$. (m) Ipomoea obscura $(70 \pm 16 \mu \mathrm{m})$. (n) Ipomoea pes-caprae subsp. brasiliensis $(151 \pm 36 \mu \mathrm{m})$. (o)-(p) Ipomoea triloba $(83 \pm 37 \mu \mathrm{m} ; 143 \pm 20 \mu \mathrm{m})$. (q) Ipomoea violacea $(170 \pm 51 \mu \mathrm{m})$. (r) Merremia gemella $(127 \pm 37 \mu \mathrm{m})$. (s) Operculina turpethum $(198 \pm 46 \mu \mathrm{m})$. (t) Stictocardia tiliifolia $(182 \pm 33 \mu \mathrm{m})$. Scale bars $=1 \mathrm{~mm}$.

1. First secondary xylem evenly divided by numerous rays..... .2

-1. First secondary xylem evenly divided by a few rays............................... .5

2. Directional successive cambium present.

Ipomoea triloba

-2. Directional successive cambium absent. 3

3. Secondary xylem with only one layer. Ipomoea nil

-3. Secondary xylem with more than one layer. . .4

4. Successive xylem with three concentric rings. Ipomoea pes-caprae subsp. brasiliensis

-4. Successive xylem dissected.... Erycibe henryi

5. Directional successive cambium present.... ...6

-5. Directional successive cambium absent............................8

6. Stem cross-section irregular. Ipomoea obscura -6. Stem cross-section with two to three lobes. .7

7. Stem cross-section with two shallow lobes. Ipomoea hederifolia -7. Stem cross-section with three deep lobes. .Merremia gemella 
8. Stem cross-section triangular........................................

-8. Stem cross-section otherwise...................................... 10

9. Cork present; first secondary xylem divided by three rays...........Ipomoea

littoralis

-9. Cork absent; first secondary xylem divided by five rays...... Stictocardia

tiliifolia

10. Secondary xylem with only one layer....................Ipomoea violacea

-10 . Secondary xylem with more than one layer.........................11

11. Stem cross-section irregular......................................... 12

-11. Stem cross-section round or oblong.............................14

12. Cork present.............................................pomoea cairica

-12. Cork absent..................................................13

13. First secondary xylem divided by two rays.................... Ipomoea indica

-13. First secondary xylem divided by three to five rays.............Ipomoea alba

14. Cork present.........................................Argyreia formsana

-14. Cork absent.......................................................15

15. Cambial arc/segment rings absent.....................Argyreia akoensis

-15. Cambial arc/segment rings present..................................16

16. Vessel diameter about $198 \pm 46 \mu \mathrm{m} . . . . . . . . . . . . . . . .$. Operculina turpethum

-16. Vessel diameter about $336 \pm 55 \mu \mathrm{m}$................. Distimate tuberosa

\section{Discussion}

In the present study, the stem of the genus Argyreia was usually round or elliptical in cross-section and the secondary xylem and secondary phloem usually formed concentric rings without rays. Parenchyma proliferation was not obvious (Figure 1). In contrast, the stem of the genus Ipomoea is irregular in cross-section, the secondary cambium rings are arcs/segments-like, and parenchyma proliferation is obvious (Figure 2(b), Figure 2(c), Figure 2(f), Figure 3(a), Figure 3(c), Figure 4(a)). The texture of the stems of Ipomoea is quite soft with irregularly distributed patches of thin-walled parenchyma, but there are almost no irregular patches of parenchyma in Argyreia. The vegetative morphologies of Argyreia and Ipomoea are quite similar, resulting in some species being erroneously described within the genus Ipomoea or Argyreia [41]. The cambial variations in their stems could be used in the debate around the species delimitation of these two genera. Vasicentric or aliform-confluent paratracheal parenchyma cells around the vessels of Argyreia and Ipomoea have been previously recorded [42], and the presence of vasicentric paratracheal parenchyma was confirmed around the vessels of the 17 species in this study.

Metaxylem elements (also called the primary xylem) differentiated from the cambium-like meristem on the primary growth were observed in these 17 species from Taiwan, and the results confirmed the metaxylem characteristics described in I. hederifolia by Lowell and Lucansky [15]. During secondary growth, the anomalous cambial stage produces the secondary xylem and secondary phloem. If the formation of secondary xylem is greater in one region than in 
another, it resulted in a grooved stem [16]. In this study, the stem cross-sections of $I$. hederifolia had two deep lobes of successive cambia. The characteristics of these lobed stems have not been described in previous reports. They might be related to stem size and the supernumerary cambial stage, and warrant further studies.

The asymmetry in the cross-sections of vines is often related to the supports used by the twining vines and results from increased tissue production in the vascular bundles. The site of the increased cambial activity is often located on the side of the stem that is not in contact with the support, e.g., in I. quamoclit [19]. In the present study, significant secondary xylem and phloem growth was observed in I. alba, I. cairica, I. indica, and I. triloba on the side of the stem opposite the support (Figure 2(b), Figure 2(c), Figure 2(f), Figure 3(e), Figure 3(f)), indicating differential cambial activity.

The eccentricity of the stem cross-sections is owing to irregular activity in the cambium causing uneven deposition of the secondary xylem. The directional intensified development of successive cambium was observed in four species, $I$. hederifolia (Figure 2(e)), I. obscura (Figure 3(c)), I. triloba (Figure 3(f)), and Merremia gemella (Figure 4(b)), along with clear, alternating xylem and phloem rings and functionally slow cambium activity on the side of the stem opposite the support. Intensified development of successive cambia can also increase stem thickness and alter the form of the pith, making it irregular, as confirmed in all the Ipomoea species, especially in I. alba, I. cairica, I. indica, I. obscura, and $I$. triloba.

The characteristics of the small arcs/segments of internal cambium in $I$. hederifolia were developed during the supernumerary cambial stage of secondary growth, as described by Lowell and Lucansky [15], Patil et al. [17], and Rajput et al. [18]. In this study, the diagnostic characteristics of the cambium arc/segment rings were present in D. tuberosa, E. henryi, I. cairica, I. obscura, I. pes-caprae subsp. brasiliensis, I. triloba, and Operculina turpethum. The species E. henryi developed more layers as adjacent segments separated by xylem rays, which would form a dissected xylem [6] in larger stems.

The supernumerary cambial stage is a common developmental stage in Ipomoea [19]. The supernumerary cambial refers to the presence of many successive rings. The stems of $I$. nil (Figure 3(b)) and I. violacea (Figure 4(a)) had only one layer of secondary xylem, whereas the stems of the remaining eight Ipomoea species possessed two to four layers of continuous xylem rings and alternating phloem rings. The stem development of the I. nil and I. violacea samples might not yet have reached the supernumerary cambial stage.

Cambial variants have been used as diagnostic characteristics for the identification of climbing plants in different families and genera, such as Aristolochiaceae, Menispermaceae, and Vitaceae. A variety of cambial variances developed in the Convolvulaceae family (Figures 1-4), as well as some climbing plants with regular secondary growth (Figures 1(a)-(f), Figure 2(a), Figure 3(d), Figure $4(\mathrm{a})$, Figure $4(\mathrm{c}))$. The development of the cambial variations in the secondary 
xylem rings did indeed differ depending on stem size (Figure 1(f), Figure 2(a)). Collecting all the different stem sizes for comparison each other are needed, and what another causes influence cambial variations in climbing plants warrant further investigation. The cambial variants of Convolvulaceae include species with unequal deposition of xylem, lobed stem formation (Figure 2(d), Figure 2(e), Figure 3(e), Figure 3(f), Figure 4(b)) that may be asymmetrical or symmetrical, intraxylary phloem, cambial arc/segment rings, and dissected xylems, all caused by the parenchyma proliferation as well as succession cambia. These variants may also be found in different combinations. Whether or not these variances have developmental relationships warrants further study.

\section{Conclusion}

Studies on the cambial variants of climbing plants in the family Convolvulaceae in Taiwan are scarce. In this study, we examined species in which cambial variants in the stem were combined with successive cambia and intraxylary phloem in Convolvulaceae from Taiwan. The experimental results showed that the stems of the two Argyreia species are round or elliptical and the secondary xylem and phloem rings are continuous. The cambial variants of Ipomoea species, except $I$. nil and $I$. violacea, include irregular conformations, successive cambia, and xylem parts dispersed by rays into segments. The first secondary xylem in E. henryi, I. nil, I. pes-caprae subsp. brasiliensis, and I. triloba is divided by numerous rays, and those of the remaining species are divided by three to five rays. The vascular bundles of E. henryi continue division and develop more layers which become adjacent segments separated by xylem rays, forming a dissected xylem. The secondary growth of $D$. tuberosa and $O$. turpethum results in only two layers of successive cambia and the second secondary xylem is divided by rays into arcs/segments. Parenchyma proliferation and mucilaginous canals distributed in the inner cortex develop noticeably in I. alba, I. cairica, and I. indica. Deeply lobed stems with directional successive cambia form irregular shapes in I. hederifolia, I. obscura, I. triloba, and M. gemella, and shallowly lobed stems form triangular shapes in I. littoralis and Stictocardia tilifolia. The successive cambia of I. pes-caprae subsp. brasiliensis forms concentric continuous bands. The xylem vessels dispersed throughout the stem are diffuse-porous and the vessels are surrounded by vasicentric paratracheal parenchyma in all the studied species. The results presented herein provide an important basis for further morphological studies on the cambial variances within this family.

\section{Acknowledgements}

We truly appreciate the assistance of the staff of the PPI Herbarium at the National Pingtung University of Science and Technology for providing fresh Convolvulaceae stem specimens in collections and photos. The authors are extremely grateful to two reviewers whose suggestions improved this manuscript. 


\section{Conflicts of Interest}

The authors declare no conflicts of interest regarding the publication of this paper.

\section{References}

[1] Mauseth, J.D. (1988) Plant Anatomy. The Benjamin/Cummings Publishing Company, Menlo Park, CA.

[2] Rajput, K.S., Chaudhary, B.D. and Patil, V.S. (2014) Development of Successive Cambia and Structure of Secondary Xylem of Ipomoea obscura (Convolvulaceae). Polish Botanical Journal, 59, 55-61. https://doi.org/10.2478/pbj-2014-0009

[3] Carlquist, S. (1975) Ecological Strategies of Xylem Evolution. University of California Press, Berkeley, CA.

[4] Carlquist, S. (2001) Comparative Wood Anatomy: Systematic, Ecological, and Evolutionary Aspects of Dicotyledon Wood. Springer-Verlag Press, Germany. https://doi.org/10.1007/978-3-662-04578-7

[5] Angyalossy, V., Angeles, G., Pace, M.R., Lima, A.C., Dias-Leme, C.L., Lohmann, L.G. and Madero-Vega, C. (2012) An Overview of the Anatomy, Development and Evolution of the Vascular System of Lianas. Plant Ecology and Diversity, 5, 167-182. https://doi.org/10.1080/17550874.2011.615574

[6] Angyalossy, V., Pace, M.R. and Lima, A.C. (2015) Liana Anatomy: A Broad Perspective on Structural Evolution of the Vascular System. In: Schnitzer, S.A., Bongers, F., Burnham, R.J. and Putz, F.E., Eds., Ecology of Lianas, John Wiley Blackwell, London, UK, 253-287. https://doi.org/10.1002/9781118392409.ch19

[7] Rajput, K.S. and Gondaliya, A.D. (2017) Internal Cambium and Intraxylary Phloem Development in Ipomoea turbinata Lag. (Convolvulaceae). Flora, 226, 47-54. https://doi.org/10.1016/j.flora.2016.11.002

[8] Rajput, K.S., Lekhakb, M.M., Kapadanec, K.K. and Yadav, S.R. (2017) Formation of Tri-lobed Stem and Development of Successive Cambia in the Stems of Argyreia hookeri C. B. Clarke (Convolvulaceae). Flora, 233, 140-149. https://doi.org/10.1016/j.flora.2017.06.005

[9] Acevedo-Rodríguez, P. (2005) Vines and Climbing Plants of Puerto Rico and the Virgin Islands. Contributions from the United States National Herbarium, 51, $1-483$.

[10] Isnard, S. and Silk, W.K. (2009) Moving with Climbing Plants from Charles Darwin's Time into the 21st Century. American Journal of Botany, 96, 1205-1221. https://doi.org/10.3732/ajb.0900045

[11] Carlquist, S. (1991) Anatomy of Vine and Liana Stems: A Review and Synthesis. In: Putz, F.E. and Mooney, H.A., Eds., The Biology of Vines, Cambridge University Press, Cambridge, UK, 53-71. https://doi.org/10.1017/CBO9780511897658.004

[12] Pant, D.D. and Bhatnagar, S. (1975) Morphological Studies in Argyreia Lour. (Convolvulaceae). Botanical Journal of the Linnean Society, 70, 45-69. https://doi.org/10.1111/j.1095-8339.1975.tb00678.x

[13] Metcalfe, C.R. and Chalk, L. (1985) Anatomy of the Dicotyledons: Volume 2: Wood Structure and Conclusion of the General Introduction, 2nd Edition, Oxford University Press, New York.

[14] Metcalfe, C.R. and Chalk, L. (1950) Anatomy of the Dicotyledons: Volume 2. Clarendon Press, Oxford. 
[15] Lowell, C. and Lucansky, T.W. (1986) Vegetative Anatomy and Morphology of Ipomoea hederifolia (Convolvulaceae). Bulletin of the Torrey Botanical Club, 113, 382-397. https://doi.org/10.2307/2996431

[16] Rajput, K.S., Raole, V.M. and Gandhi, D. (2008) Radial Secondary Growth and Formation of Successive Cambia and Their Products in Ipomoea hederifolia L. (Convolvulaceae). Botanical Journal of the Linnean Society, 158, 30-40. https://doi.org/10.1111/j.1095-8339.2008.00854.x

[17] Patil, V.S., Rao, K.S. and Rajput, K.S. (2009) Development of Intraxylary Phloem and Internal Cambium in Ipomoea hederifolia (Combretaceae). The Journal of the Torrey Botanical Society, 136, 423-432. https://doi.org/10.3159/09-RA-033.1

[18] Rajput, K.S., Patil, V.S. and Rao, K.S. (2014) Multiple Cambia and Secondary Xylem of Ipomoea pes-caprae (L.) R. Br. (Convolvulaceae). Acta Botanica Gallica, 161, 13-19. https://doi.org/10.1080/12538078.2013.847020

[19] Lowell, C. and Lucansky, T.W. (1990) Vegetative Anatomy and Morphology of Ipomoea quamoclit (Convolvulaceae). Bulletin of the Torrey Botanical Club, 117, 232-246. https://doi.org/10.2307/2996692

[20] Christenhusz, M.J.M. and Byng, J.W. (2016) The Number of Known Plants Species in the World and Its Annual Increase. Phytotaxa, 261, 201-217. https://doi.org/10.11646/phytotaxa.261.3.1

[21] Staples, G.W. and Yang, S.Z. (1998) Convolvulaceae. In: Huang, T.C., et al., Eds., Flora of Taiwan, 2nd Edition, Department of Botany National Taiwan University, Taipei, 341-384.

[22] Yang, S.Z., Chen, P.H., and Staples, G.W. (2015) Argyreia akoensis (Convolvulaceae), a New Species from Southern Taiwan. Taiwania, 60, 117-122.

[23] Liao, G.I., Chen, M.Y. and Kuoh, C.S. (2000) Cuscuta L. (Convolvulaceae) in Taiwan. Taiwania, 45, 226-234.

[24] Chen, S.H. and Wu, M.J. (2001) Notes on Two Newly Naturalized Plants in Taiwan. Taiwania, 46, 85-92.

[25] Hsu T.W., Chiang, T.Y., Tsai, K.C. and Huang, C.C. (2006) Ipomoea eriocarpa R. Br. (Convolvulaceae) Newly Naturalized to Taiwan. Taiwan Journal of Biodiversity, 8, 103-107.

[26] Hsu, T.W., Wang, K.H. and Chiang, T.Y. (2006) Ipomoea purpurea (L.) Roth (Convolvulaceae), A Species of the I. nil Complex, Newly Naturalized in Taiwan. BioFormosa, 41, 19-22.

[27] Chen, P.H. and Yang, S.Z. (2017) Ipomoea leucantha Jacq. (Convolvulaceae), a Newly Naturalized Plant in Taiwan. Journal of the National Taiwan Museum, 70, $1-8$.

[28] Chao, C.T., Cheng, C.L. and Wang, C.M. (2019) Ipomoea lacunosa (Convolvulaceae: Ipomoea sect. Batatas): A Newly Naturalized Species of Taiwan. Taiwan Journal of Forestry Science, 34, 55-62.

[29] Chen, S.H., Su, J.Y. and Wu, M.J. (2009) Notes on Two Newly Naturalized Plants in Taiwan: Evolvulus nummularius (L.) L. (Convolvulaceae) and Acalypha aristata Kunth (Euphorbiaceae). Taiwania, 54, 273-278.

[30] Tsai Y.C., Lee, P.J., Chang, Y.H. and Hsieh, T.H. (2010) Merremia dissecta (Jacq.) H. Hallier., a Newly Naturalized Species in Taiwan. Journal of Ecology and Environmental Sciences, 3, 1-7.

[31] Ko, Y.N. and Liu, H.Y. (2011) Merremia cissoides (Lam.) Hallier f. (Convolvulaceae), a Newly Naturalized Plant to Taiwan. Taiwan Journal of Biodiversity, 13, 
345-348.

[32] Chung, S.W., Hsu, T.C. and Chung, A.C. (2017) Merremia quinquefolia (Convolvulaceae), a Newly Naturalized Plant in Taiwan. Taiwan Journal of Biodiversity, 19, 123-129.

[33] Chao, C.T., Chen, P.H. and Wang, C.M. (2017) Two Newly Naturalized Plant Species in Taiwan: Astraea lobata and Merremia umbellata. Quarterly Journal of Forest Research, 39, 285-294.

[34] Yang, S.Z. and Buril, M.T. (2012) A New Alien Distribution Record and Addition to the Flora of Taiwan: Jacquemontia polyantha (Schltdl. \& Cham.) Hallier f. (Convolvulaceae). Taiwania, 57, 418-421.

[35] Yang, S.Z. and Chen, P.H. (2016) Cambial Variants in the Family Menispermaceae in Taiwan. American Journal of Plant Science, 7, 841-854. https://doi.org/10.4236/ajps.2016.76080

[36] Yang, S.Z., Chen, P.H. and Lin, K.C. (2016) Cambial Variants of Liana Species (Fabaceae) in Taiwan. Taiwania, 61, 185-193.

[37] Simões, A.R. and Staples, G. (2017) Dissolution of Convolvulaceae Tribe Merremieae and a New Classification of the Constituent Genera. Botanical Journal of the Linnean Society, 183, 561-586. https://doi.org/10.1093/botlinnean/box007

[38] Radford, A.E. (1986) Fundamentals of Plant Systematics. Harper \& Row Publishers, Inc., New York.

[39] Wheeler, E.A., Baas, P. and Gasson, P.E. (1989) IAWA List of Microscopic Features for Hardwood Identification with an Appendix on Non-anatomical Information. IA WA Bulletin, 10, 219-332. https://doi.org/10.1163/22941932-90000496

[40] Ferreira, T. and Rasband, W. (2011) The ImageJ User Guide Version 1.44. http://rsbweb.nih.gov/ij/docs/user-guide.pdf

[41] Fang, R.Z. and Staples, G.W. (1995) Convolvulaceae. In: Wu, Z.Y. and Raven, P.H., Eds., Flora of China, Science Press, Beijing, and Missouri Botanical Garden Press, Saint Louis, MO, 271-327.

[42] Carlquist, S. and Hanson, M.A. (1991) Wood and Stem Anatomy of Convolvulaceae: A Survey. Aliso: A Journal of Systematic and Evolutionary Botany, 13, 51-94. https://doi.org/10.5642/aliso.19911301.03 